\title{
Association between toxocariasis and cognitive function in young to middle-aged adults
}

\author{
${ }^{1}$ Department of Sociology, Brigham Young University, Provo, Utah, USA; \\ ${ }^{2}$ Department of Psychology, Brigham Young University, Provo, Utah, USA; \\ ${ }^{3}$ The Neuroscience Center, Brigham Young University, Provo, Utah, USA
}

Lance D. Erickson ${ }^{1}$, Shawn D. Gale ${ }^{2,3}$, Andrew Berrett ${ }^{2}$, Bruce L. Brown ${ }^{2}$ and Dawson W. Hedges ${ }^{2,3}$

\begin{abstract}
The ascarid nematodes Toxocara canis (Werner, 1782) and Toxocara cati (Schrank, 1788) may infect humans resulting in toxocariasis. A prior study associated species of Toxocara Stiles, 1905 with cognitive deficits in children. To determine if a similar association between toxocariasis and cognition exists in adults, we analysed a large dataset from the United States' Center for Disease Control's National Health and Nutrition Examination Survey. We used linear-regression and multivariate models to examine the association between toxocariasis as assessed by the presence of anti-Toxocara IgG antibodies and three measures of cognitive function - simple reaction time (SRT), symbol-digit substitution (SDS) and serial-digit learning (SDL) in 4279 adults aged 21 to 59 years. Toxocara seroprevalence did not vary with age or blood-lead concentration but did vary with gender, ethnicity, educational attainment and poverty-to-income ratio. Controlling for gender, age, blood-lead concentration, educational attainment, ethnic background and the poverty-to-income ratio, we found that toxocariasis predicted worse performance on the SDS but not on the SRT or the SDL. Moreover, there were significant interactions between toxocariasis and age, gender and educational attainment. In conclusion, toxocariasis appears to be associated with decreased cognitive function. Interactions between toxocariasis and gender, age and educational attainment further suggest that certain groups may be more susceptible than others to the cognitive dysfunction associated with toxocariasis in adults.
\end{abstract}

Keywords: cognition, Third National Health and Nutrition Examination Survey (NHANES III), Toxocara canis, Toxocara cati

Accumulating evidence suggests that infectious diseases can be associated with impaired cognitive function (Aiello et al. 2006, Katan et al. 2013) including dementia (Malaguarnera et al. 2004, Kountouras et al. 2006, Kusbeci et al. 2011, Lurain et al. 2013). To date, Helicobacter pylori, Chlamydia pneumoniae, cytomegalovirus and herpes types 1 and 2 have been found to be associated with decreased cognitive function in older adults (Kusbeci et al. 2011, Katan et al. 2013). In a sample ranging in age from 20 to 90 years, Helicobacter pylori infection was associated with lower cognitive ability (Beydoun et al. 2013).

In addition to chronic infection from viruses and bacteria, parasitic infections have also been associated with cognitive function. For example, latent infection with Toxoplasma gondii (Nicolle et Manceaux, 1908), an intracellular apicomplexan parasite, has been associated with cognitive impairment (Havlíček et al. 2001, Gale et al. 2015).

Caused by the ascarid nematodes Toxocara canis (Werner, 1782) and Toxocara cati (Schrank, 1788), toxocariasis can be transmitted to humans via eggs in dog and cat faeces after embryonation in soil or on vegetables, although there is also evidence that exposure to infected, undercooked meat may also result in infection (Sturchler et al. 1990,
Despommier 2003, Fillaux and Magnaval 2013). Species of Toxocara Stiles, 1905 do not reproduce in humans, but larvae migrate throughout the body, including the brain (Despommier 2003), and infection can result in chronically elevated antibody titres (Lee et al. 2014). Toxocara canis and Toxocara cati are found worldwide (Despommier 2003). In the United States, the overall seroprevalence of toxocariasis is estimated at approximately 14 percent, although considerable variation exists between ethnic groups (Walsh and Haseeb 2012).

Inbred mice infected with $T$. canis showed diminished learning ability (Hamilton et al. 2006). Similarly, rats experimentally infected with toxocariasis showed changes in open-field behaviour (Chieffi et al. 2010). In humans, Marmor et al. (1987) found small differences in performance between case-control pairs in 155 children seropositive for $T$. canis. In a more recent, larger, and better controlled study that included data from a national survey in the United States conducted by the Centers for Disease Control, Walsh and Haseeb (2012) found that children seropositive for Toxocara antibodies performed more poorly on standard tasks measuring attention and perceptual reasoning and on two measures of academic achievement compared to se- 
ronegative children while controlling for gender, ethnicity, blood-lead levels and socioeconomic status.

Given the findings of widespread human exposure to toxocariasis, the reported associations between cognition in mice and human children, the potential for prevention and the lack of studies evaluating the potential effect of toxocariasis on cognition in human adults (Holland and Hamilton 2013), we sought to investigate the effects of latent infection with toxocariasis on cognitive function in young to middle-aged adults.

\section{MATERIALS AND METHODS}

\section{Study sample}

We analysed publically available anonymised data from the Third National Health and Nutrition Examination Survey (NHANES III) carried out from 1988-1994 by the National Center for Health Statistics at the Centers for Disease Control Centers for Disease Control and Prevention (CDC; Centers for Disease Control and Prevention 2007). This was the most recent NHANES III dataset containing the relevant variables by which to evaluate the association between toxocariasis and cognition in adults. The NHANES III sample is designed to be representative of the United States' population. These data were collected by the U.S. government in accordance with federal guidelines associated with ethical conduct in research.

\section{Cognitive function}

As detailed elsewhere (Krieg et al. 2001), cognitive function in NHANES III for adults aged 21 to 59 years was evaluated via computer-administered tests of cognitive function. The three measures of cognitive function available in the NHANES III dataset for adults were simple reaction time (SRT), symbol-digit substitution (SDS) and serial-digit learning (SDL). Prior studies have demonstrated adequate psychometric properties of these tests including reliability, validity and sensitivity to cognitive function (Baker et al. 1985, Arcia and Otto 1992, Krieg et al. 2001). The SRT is a measure of reaction time obtained via pressing a keyboard button when a stimulus appears on the computer screen. Presentation of stimuli varies randomly between 2500 and $5000 \mathrm{~ms}$. The SDS is a coding task in which numbers and symbols are paired. Using a key located at the top of the screen, the subject views symbols at the bottom of the screen and types in each symbol's corresponding number onto the keyboard. Performance is measured in seconds (latency) and each participant completed five trials, one of which was a practice trial. The summary score for this measure was based on the average of the two lowest (i.e. fastest) error-corrected latencies. Thus, the score is computed by dividing the time (latency) in seconds by the total number of correct responses (seconds/number of correct coded pairs). In the SDL, a series of digits is viewed on the computer screen and then the subject must type in the numbers from the series onto the keyboard. Performance represents the number of trials needed to reach complete two consecutive trials without error. Accordingly, for each of the cognitive tests, a lower score equated to better performance. Notably, the cognitive tests administered to adults differed from those administered to children enrolled in the NHANES III survey, precluding direct comparison between the toxocariasis study in children (Walsh and Haseeb 2012) and the present study. Specifically, the cognitive data collected in children for NHANES III was limited to the reading and math subtests from the Wide Range Achievement Test-Revised (WRAT-R), a measure of academic achievement, and two subtests from the Wechsler Intelligence Scale for Children-Revised (WISC-R), a measure of intellectual function. Since the WRAT-R and WISC-R were not given to adults as part of the national NHANES III survey, we cannot compare scores between children and adults.

\section{Toxocara spp.}

Diagnosis of toxocariasis was determined by antibody testing for Toxocara canis using a CDC-developed indirect enzyme immunoassay. An excretory/secretory antigen of $T$. canis is reacted with test serum and then anti-human IgG. Because this assay reacted to both $T$. canis and $T$. cati, we were unable to differentiate between T. canis and T. cati (see Won et al. 2008). The addition of tetra-methylbenzidine substrate allows for optical density analysis, thereby determining the presence or absence of antibodies against Toxocara, as detailed further in the relevant documentation provided by the CDC (Centers for Disease Control and Prevention 2007). The NHANES III database does not include antibody titre levels; instead, the assay results are reported as positive (1) or negative (0) for infection with Toxocara. Thus, we included toxocariasis as a dichotomous categorical variable in the predictive model and were unable to categorise titre levels or report serointensity.

\section{Demographic covariates}

In an effort to address potential confounding variables as well as allow for the investigation of potential interactions with Toxocara, we used a number of demographic covariates in the models. Continuous variables included education, age in years, and poverty-to-income ratio (PIR). Education level was determined by the number of years of schooling achieved. Therefore, larger numbers indicate higher levels of education. PIR is defined as the ratio between total family income and the United States poverty threshold (at the time of the administration of the NHANES III). A value above one indicates an income above poverty level. Based on preliminary analysis, the relationship between PIR and each outcome was modelled as a spline including two separate but connecting regression lines because it had two distinct linear components with the knot or deflection point at PIR of three. Race-ethnicity and gender were modelled as categorical covariates. Race-ethnicity categories were grouped into non-Hispanic white, non-Hispanic black, Hispanic and other (including Aleut, Eskimo, American Indian, Asian or Pacific Islander and other). Finally, gender was coded zero for male and one for female. The combination of education and PIR provides an estimate of overall socioeconomic status (Suresh et al. 2011) and it is for this reason that we included both in our model. In addition to these demographic variables and based on the association between lead exposure in human adults and cognition (Schwartz et al. 2000) and the findings of Walsh and Haseeb (2012) showing higher blood-lead concentrations in children positive for Toxocara than in children negative for Toxocara, we included the blood-lead concentrations reported in the NHANES III dataset as a control variable. 
Table 1. Weighted prevalence of anti-Toxocara antibodies across demographic characteristics in USA 21 to 59 year-olds, Third National Health and Nutrition Examination Survey (NHANES III) (1988-1994).

\begin{tabular}{|c|c|c|c|c|}
\hline & $\mathrm{N}^{\mathrm{a}}$ & $\%$ Toxocara $[95 \% \mathrm{CI}]^{\mathrm{b}}$ & Odds ratio ${ }^{c}$ & $p$-value ${ }^{\mathrm{d}}$ \\
\hline Age & & & & 0.068 \\
\hline $21-30$ & 1313 & $14.4[11.15,17.58]$ & 0.88 & \\
\hline $31-40$ & 1218 & $17.1[13.53,20.62]$ & 1.08 & \\
\hline $41-50$ & 1022 & $12.2[9.10,15.38]$ & 0.73 & \\
\hline $51-59$ & 726 & $11.2[6.94,15.47]$ & 0.66 & \\
\hline Gender & & & & 0.045 \\
\hline Male & 2041 & $15.9[12.77,19.08]$ & 0.99 & \\
\hline Female & 2238 & $12.2[9.93,14.50]$ & 0.73 & \\
\hline Race ethnicity & & & & 0.029 \\
\hline Non-hispanic white & 1575 & $13.0[10.42,15.52]$ & 0.78 & \\
\hline Non-hispanic black & 1300 & $21.0[18.89,23.14]$ & 1.39 & \\
\hline Hispanic & 1249 & $12.8[10.48,15.14]$ & 0.77 & \\
\hline Other & 155 & $17.6[7.71,27.43]$ & 1.12 & \\
\hline Education & & & & $<0.001$ \\
\hline No high school diploma & 1378 & $23.0[18.29,27.74]$ & 1.57 & \\
\hline High school diploma & 1433 & $13.5[10.52,16.48]$ & 0.82 & \\
\hline More than high school & 1468 & $10.9[8.39,13.33]$ & 0.64 & \\
\hline Poverty ${ }^{e}$ & & & & $<0.001$ \\
\hline Not in poverty (i.e. > 1) & 3264 & $12.0[10.99,14.85]$ & 0.72 & \\
\hline In poverty $($ i.e. $\leq 1)$ & 1015 & $22.4[17.75,27.06]$ & 1.52 & \\
\hline Blood lead $(\mu \mathrm{g} / \mathrm{dl})^{\mathrm{f}}$ & & & & 0.082 \\
\hline $0.0-1.9$ & 1443 & $12.1[9.40,14.70]$ & 0.72 & \\
\hline $2.0-3.8$ & 1439 & $15.5[12.63,18.45]$ & 0.96 & \\
\hline $3.9-42.0$ & 1397 & $15.1[12.07,18.05]$ & 0.93 & \\
\hline
\end{tabular}

$\mathrm{N}=4279$; ${ }^{\mathrm{a}}$ numbers are unweighted numbers of participants; ${ }^{\mathrm{b}}$ prevalence percentages are weighted to be representative of the U.S. population. Toxocara-positive defined by the presence of anti-Toxocara antibodies; ${ }^{c}$ the reference odds for constructing the odds ratio is the estimated $16 \%$ overall seroprevalence of Toxocara in this NHANES III dataset; ${ }^{\mathrm{d}} p$ based on Pearson's $\chi^{2}$ test, which tests whether the prevalence of toxocariasis differs across

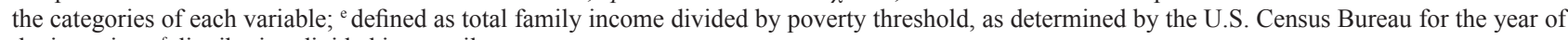
the interview; ${ }^{\mathrm{f}}$ distribution divided into tertiles.

\section{Statistical analyses}

We used Stata 13.1 (StataCorp, College Station, Texas) for all statistical analyses. Analyses were performed using Stata's svy command prefix, which allows for the inclusion of sampling weights to adjust parameter estimates to be representative of the U.S. civilian non-institutionalised population aged 21 to 59 years as well as adjustments to standard-error estimates to account for the complex-sampling (i.e. stratified) design. Pearson's $\chi^{2}$ tests are presented in Table 1 to compare the prevalence of species of Toxocara across values of the independent variables.

Since we used three different dependent variable measures of cognitive functioning as predicted by both categorical and continuous independent variables, we used MANCOVA, which enabled the covariances of the dependent variables to be taken into account in a multivariate test of the relationship between toxocariasis and the three cognitive outcome variables. Because the traditional MANCOVA procedures available in commonly available statistical packages cannot handle the complex-sampling nature of the NHANES III data (i.e. non-random, stratified and clustered sampling), it was necessary to use maximum likelihood estimation methods to calculate the multivariate hypothesis tests.

Typically, the output of MANCOVA analyses includes both the holistic multivariate analysis results and the corresponding univariate results for each dependent variable. We accomplished the univariate tests of the hypotheses for each of the dependent variables separately using Ordinary Least Squares models. The multivariate hypothesis tests of the holistic pattern of effects of the independent variables of all three dependent variables simultaneously were accomplished using Stata's maximum likelihood suest command. This command combines the estimation results, including parameter estimates for individual variables and also including covariance parameter estimates, into a single parameter vector. The results of this multivariate test are expressed in a single $\mathrm{F}$ value rather than the four usual multivariate statistics (Wilks' lambda, Hotelling-Lawley trace, Pillai's trace, and Roy's largest root).

We completed the analyses in two stages. First, we predicted cognitive functioning as measured by the SDS, SRT, and SDL using toxocariasis as the main predictor and controlling for age, gender, race-ethnicity, education, PIR and blood-lead concentration. Second, we estimated a series of two-way interaction models, each including an interaction between toxocariasis and a single covariate and with the remaining controls. In other words, we estimated models for the interaction between toxocariasis and age, toxocariasis and gender, toxocariasis and race-ethnicity, and so on.

\section{RESULTS}

The overall seroprevalence of Toxocara canis and T. cati (referred to as Toxocara) in this sample was $16 \%$. The weighted seroprevalence of toxocariasis across values of the control variables is presented in Table 1. Males were more likely to have toxocariasis than females $(15.9 \%$ versus $12.2 \%, p=0.045)$. While there were no significant differences in seroprevalence between age groups $(p=0.068)$, seroprevalence varied among race-ethnic groups ( $p=0.029)$, with non-Hispanic blacks having a seroprevalence of $21.0 \%$, compared to $12.8 \%$ for Hispanics 
Table 2. Multivariate analysis ${ }^{\mathrm{a}}$ of cognitive functioning in USA 21 to 59 year-olds, Third National Health and Nutrition Examination Survey (1988-1994): unstandardised coefficients from weighted Ordinary Least Squares regression.

\begin{tabular}{lccc}
\hline & SDS & SDL & SRT \\
\hline $\begin{array}{l}\text { Constant } \\
\text { Toxocara }\end{array} \quad 3.07[2.85,3.30]$ & $8.44[7.04,9.85]$ & $249.71[233.55,265.88]$ \\
$\quad$ Negative & $0.00[0.00,0.00]$ & $0.00[0.00,0.00]$ & $0.00[0.00,0.00]$ \\
$\quad$ Positive & $0.12[0.02,0.22]$ & $0.48[-0.01,0.96]$ & $0.28[-4.63,5.20]$ \\
Age & $0.03[0.03,0.03]$ & $0.10[0.08,0.11]$ & $0.50[0.28,0.72]$ \\
Gender & & & \\
$\quad$ Male & $0.00[0.00,0.00]$ & $0.00[0.00,0.00]$ & $0.00[0.00,0.00]$ \\
Female & $-0.14[-0.20,-0.08]$ & $-0.04[-0.36,0.28]$ & $10.68[5.71,15.65]$ \\
Race ethnicity & & & \\
$\quad$ Non-hispanic white & $0.00[0.00,0.00]$ & $0.00[0.00,0.00]$ & $0.00[0.00,0.00]$ \\
Non-hispanic black & $0.36[0.29,0.43]$ & $1.62[1.24,2.01]$ & $12.30[6.76,17.84]$ \\
Hispanic & $0.26[0.16,0.37]$ & $2.30[1.72,2.88]$ & $7.95[1.89,14.00]$ \\
$\quad$ Other & $0.30[0.14,0.46]$ & $2.21[1.20,3.23]$ & $1.11[-6.29,8.52]$ \\
Highest grade achieved & $-0.11[-0.12,-0.09]$ & $-0.52[-0.59,-0.44]$ & $-2.37[-3.36,-1.37]$ \\
Poverty-to-income ratio & & & \\
$\quad 0-3$ & $-0.11[-0.16,-0.06]$ & $-0.53[-0.85,-0.21]$ & $-3.17[-6.03,-0.31]$ \\
3-11 & $-0.00[-0.03,0.02]$ & $-0.14[-0.31,0.03]$ & $-1.89[-3.78,-0.01]$ \\
Blood lead $(\mu \mathrm{g} / \mathrm{dl})$ & $0.01[-0.00,0.02]$ & $-0.02[-0.11,0.07]$ & $-0.96[-1.68,-0.23]$ \\
\hline
\end{tabular}

Abbreviations: SDL - serial digit learning test; $\mathrm{SRT}$ - simple reaction time test; SDS - symbol digit substitution test. Notes: $\mathrm{N}=4279 ;{ }^{\mathrm{a}}$ the multivariate statistical test is presented in the text; ${ }^{\mathrm{b}}$ Toxocara-positive defined by the presence of anti-Toxocara antibodies; ${ }^{\mathrm{c}}$ defined as total family income divided by poverty threshold, as determined by the U.S. Census Bureau for the year of the interview.

Table 3. Schematic table of significant two-way interactions between Toxocara and model variables and associated $p$-values from multivariate tests.

\begin{tabular}{|c|c|c|c|c|}
\hline & $p$-values from multivariate tests & Dependent variable 1 (SDS) & Dependent variable 2 (SDL) & Dependent variable 3 (SRT) \\
\hline Age & 0.011 & - & - & $x$ \\
\hline Female & 0.034 & - & $x$ & - \\
\hline \multicolumn{5}{|l|}{ Race ethnicity } \\
\hline Non-hispanic black & 0.758 & - & - & - \\
\hline Hispanic & 0.843 & - & - & - \\
\hline Other & 0.307 & - & - & - \\
\hline Highest grade achieved & 0.001 & $x$ & $x$ & - \\
\hline \multicolumn{5}{|l|}{ Poverty-to-income ratio } \\
\hline $0-3$ & 0.178 & - & - & - \\
\hline $3-11$ & 0.070 & - & - & - \\
\hline
\end{tabular}

Abbreviations: SDL - Serial digit learning test; SRT - Simple reaction time test; SDS - Symbol digit substitution test. Notes: N = 4279; only significant univariate tests that were protected from alpha inflation by a significant multivariate test are reported; cells represent the interaction between the variable represented by the row and the column. For example, the $\times$ at the intersection of the Female row and the SDL column indicates a significant interaction between Toxocara and gender.

and $13.0 \%$ for non-Hispanic whites. Seroprevalence declined with increasing educational attainment $(p<0.001)$ and was higher among those in poverty (PIR $>1=12.0 \%$ compared to PIR $\leq 1=22.4 \%, p<0.001)$ but was unrelated to blood-lead levels $(p=0.082)$.

Table 2 reports results from the first part of the analysis - the unstandardised coefficients and confidence intervals for the univariate tests of the relationship between Toxocara and the three cognitive functioning outcomes. Since we are interested only in the toxocariasis coefficient here with the other variables only being controls, we estimate a single multivariate test for the relationship of toxocariasis and the three cognitive function outcomes. The multivariate test was significant $(F(3,47)=2.81, p=0.0495)$, driven primarily by the relationship between toxocariasis and the SDS $(b=0.12 ; 95 \% \mathrm{CI}, 0.02,0.22)$. Generally, the unstandardised coefficients represent the predicted average change in the dependent variable for a one-unit increase in the independent variable. In this case, those with Toxo- cara exposure had an average SDS score (ratio of seconds/ number correct) 0.12 higher than those without toxocariasis while controlling for age, gender, race-ethnicity, educational attainment, PIR and blood-lead concentration. Higher scores reflect worse performance. The average ratio across the entire sample for the SDS was 2.9 and the standard deviation was 1.1.

Table 3 summarises the results from the second part of the analysis that examines whether the relationship between Toxocara and cognitive functioning differs for different values of the demographic covariates. The first column presents $p$-values from the multivariate tests that examine each interaction with Toxocara. For instance, the first row represents the interaction between age and Toxocara and is multivariately significant $(F(3,47)=4.12$, $p=0.011)$. Female $(F(3,47)=3.13, p=0.034)$ and highest grade achieved $(F(3,47)=7.56, p=0.001)$ also had interactions with cognitive functioning that were multivariately significant. The remaining rows show the significant uni- 
Table 4. Two-way interaction effect of Toxocara and education ${ }^{\mathrm{a}}$ on symbol digit substitution in USA 21 to 59 year-olds, Third National Health and Nutrition Examination Survey (1988-1994): unstandardised coefficients from weighted Ordinary Least Squares regression.

\begin{tabular}{lc}
\hline & $\begin{array}{c}\text { Interaction with } \\
\text { education }\end{array}$ \\
\hline Constant & $2.99[2.77,3.21]$ \\
Toxocara & \\
Negative & $0.00[0.00,0.00]$ \\
Positive & $0.54[0.11,0.96]$ \\
Age & $0.03[0.03,0.03]$ \\
Gender & \\
Male & $0.00[0.00,0.00]$ \\
Female & $-0.14[-0.20,-0.08]$ \\
Race ethnicity & \\
$\quad$ Non-hispanic white & $0.00[0.00,0.00]$ \\
Non-hispanic black & $0.37[0.30,0.44]$ \\
Hispanic & $0.27[0.17,0.37]$ \\
Other & $0.30[0.15,0.45]$ \\
Highest grade achieved & $-0.10[-0.12,-0.09]$ \\
Poverty-to-income ratio & \\
0-3 & $-0.11[-0.15,-0.06]$ \\
3-11 & $-0.00[-0.03,0.02]$ \\
Blood lead $(\mu \mathrm{g} /$ dl) & $0.01[-0.00,0.02]$ \\
Interactions with Toxocara & \\
Highest grade achieved & $-0.03[-0.06,-0.00]$ \\
\hline
\end{tabular}

Notes: $\mathrm{N}=4279 ;{ }^{\text {a }}$ other interactions between Toxocara and control variables were not significant; ${ }^{\mathrm{b}}$ Toxocara-positive defined by the presence of anti-Toxocara antibodies; ${ }^{\circ}$ defined as total family income divided by poverty threshold, as determined by the U.S. Census Bureau for the year of the interview.

Table 6. Two-way interaction effect of Toxocara and age ${ }^{a}$ on simple reaction time in USA 21 to 59 year-olds, Third National Health and Nutrition Examination Survey (1988-1994): unstandardised coefficients from weighted Ordinary Least Squares regression.

\begin{tabular}{lc}
\hline & Interaction with age \\
\hline Toxocara & \\
Negative & $0.00[0.00,0.00]$ \\
Positive & $16.27[4.26,28.28]$ \\
Age & $0.56[0.32,0.80]$ \\
Gender & \\
$\quad$ Male & $0.00[0.00,0.00]$ \\
Female & $10.72[5.77,15.66]$ \\
Race & \\
Non-hispanic white & $0.00[0.00,0.00]$ \\
Non-hispanic black & $12.31[6.76,17.87]$ \\
Hispanic & $7.87[1.83,13.91]$ \\
Other & $1.06[-6.31,8.42]$ \\
Highest grade achieved & $-2.36[-3.35,-1.38]$ \\
Poverty-to-income ratio & \\
0-3 & $-3.16[-6.03,-0.28]$ \\
3-11 & $-1.89[-3.78,0.00]$ \\
Blood lead $(\mu \mathrm{g} /$ dl) & $-.96[-1.68,-0.24]$ \\
Interactions with Toxocara & \\
Age & $-0.44[-0.74,-0.13]$ \\
Constant & $247.38[230.66,264.09]$ \\
\hline
\end{tabular}

Notes: $\mathrm{N}=4279$; ${ }^{\text {a }}$ other interactions between Toxocara and control variables were not significant; ${ }^{\mathrm{b}}$ Toxocara-positive defined by the presence of anti-Toxocara antibodies; ${ }^{c}$ defined as total family income divided by poverty threshold, as determined by the U.S. census bureau for the year of the interview.
Table 5. Two-way interaction effects of Toxocara with gender and education ${ }^{\mathrm{e}}$ on serial digit learning in USA 21 to 59 yearolds, Third National Health and Nutrition Examination Survey (1988-1994): unstandardised coefficients from weighted Ordinary Least Squares regression.

\begin{tabular}{lcc}
\hline & $\begin{array}{c}\text { Interaction with } \\
\text { gender }\end{array}$ & $\begin{array}{c}\text { Interaction with } \\
\text { education }\end{array}$ \\
\hline Constant & $8.33[6.93,9.72]$ & $7.99[6.55,9.43]$ \\
Toxocara & $.00[.00, .00]$ & $.00[.00, .00]$ \\
Negative & $1.11[.32,1.90]$ & $2.83[1.20,4.47]$ \\
Positive & $.10[.08, .11]$ & $.10[.08, .11]$ \\
Age & & \\
Gender & $.00[.00, .00]$ & $.00[.00, .00]$ \\
Male & $.15[-.21, .52]$ & $-.04[-.36, .28]$ \\
Female & $.00[.00, .00]$ & $.00[.00, .00]$ \\
Race ethnicity & $1.63[1.25,2.01]$ & $1.65[1.27,2.03]$ \\
Non-hispanic white & $2.33[1.74,2.91]$ & $2.33[1.75,2.91]$ \\
Non-hispanic black & $2.20[1.20,3.21]$ & $2.20[1.19,3.20]$ \\
Hispanic & $-.52[-.59,-.44]$ & $-.49[-.56,-.41]$ \\
Other & & \\
Highest grade achieved & $-.54[-.85,-.22]$ & $-.52[-.84,-.21]$ \\
Poverty-to-income ratio & $-.14[-.31, .03]$ & $-.15[-.32, .02]$ \\
0-3 & $-.02[-.11, .06]$ & $-.02[-.11, .07]$ \\
3-11 & & \\
Blood lead $(\mu \mathrm{g} /$ dl) & $.00[.00, .00]$ & \\
Interactions with Toxocara & $-1.49[-2.63,-.34]$ & \\
Gender & & $-.19[-.31,-.07]$ \\
Male & & \\
Female & & \\
Highest grade achieved & & \\
\hline
\end{tabular}

Notes: $\mathrm{N}=4279$; ${ }^{a}$ other interactions between Toxocara and control variables were not significant; ${ }^{b}$ Toxocara-positive defined by the presence of anti-Toxocara antibodies; ${ }^{c}$ defined as total family income divided by poverty threshold, as determined by the U.S. Census Bureau for the year of the interview.

variate interactions that were accompanied by a significant multivariate test. For instance, there was not a significant interaction between age and Toxocara for SDS or SDL but there was a significant, negative interaction for SRT. The significant multivariate test for female was driven by the age and Toxocara interaction on SDL and there were significant univariate interactions between highest grade achieved and Toxocara on SDS and SDL.

Although all significant interactions had negative signs, it is difficult to obtain an intuitive sense for the form of the interactions just by the coefficients' signs (Table 2) or even their magnitude. Tables 4-6 present the coefficients for each of the univariate models of SDS, SDL and SRT, respectively, that had a significant interaction between a demographic covariate and Toxocara, and where the accompanying multivariate test was significant. The interactions themselves are characterised jointly by the main effects (the coefficients for the control and Toxocara) and the interaction term (the product of the control and Toxocara), which become much more intuitive if predictions from the models are estimated.

Consequently, we present predictions from these models in Figs. 1-3. Figure 1 shows that males with Toxocara have higher SDL scores (i.e. worse cognitive functioning) than males without Toxocara but that, although the difference is small, females with Toxocara have slightly lower SDL scores (i.e. better cognitive functioning). Figure 2 shows an 


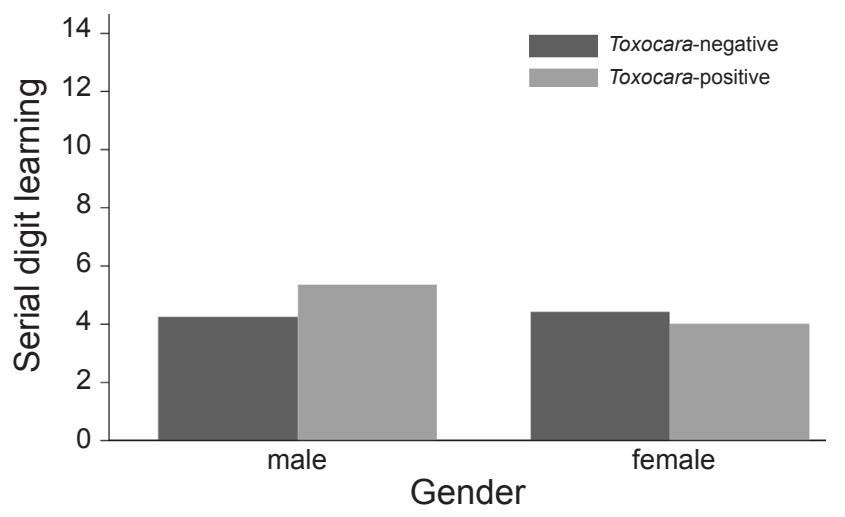

Fig. 1. The interaction of Toxocara and sex on serial digit learning: predicted values from Ordinary Least Squares Regression. Note: Predictions from the 'interaction with gender' model of serial digit learning reported in Table 5.
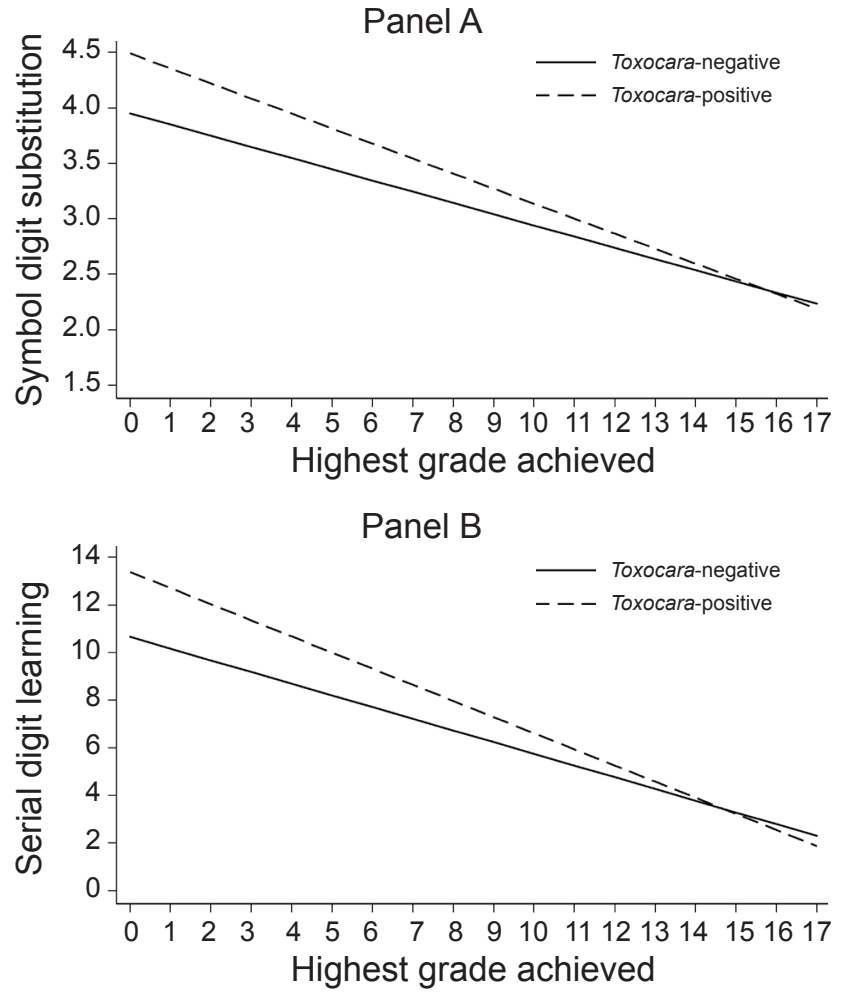

Fig. 2. The interaction of Toxocara and highest grade achieved on symbol digit substitution (Panel A) and serial digit learning (Panel B): predicted values from Ordinary Least Squares regression. Note: Predictions for Panel A come from the 'Interaction with Education' model of Symbol Digit Substitution reported in Table 4; predictions for Panel B come from the 'Interaction with Education' model of serial digit learning reported in Table 5.

almost identical pattern for the interaction between highest grade achieved and Toxocara on SDS and SDL. At lower levels of education, those Toxocara-positive have worse cognitive functioning, but as education increases, the difference between those Toxocara-positive and negative decreases and disappears. In other words, education appears to be protective against the cognitive effects of Toxocara. Finally, Fig. 3 shows that SRT scores increase with age (i.e. worse cognitive functioning) among those that are Toxo-

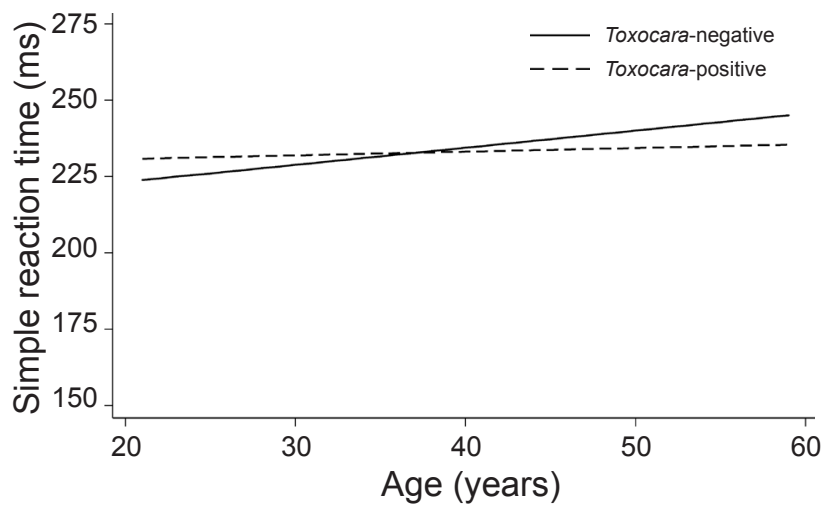

Fig. 3. The interaction of Toxocara and age on simple reaction time: Predicted values from Ordinary Least Squares regression. Note: Predictions from 'Interaction with age' model of simple reaction time reported in Table 6.

cara-negative, but that the relationship between age and SRT is almost 0 among those that are Toxocara-positive. In other words, Toxocara appeared to be associated with better performance rather than cognitive decline when cognitive function was measured by SRT.

\section{DISCUSSION}

The overall prevalence of anti-Toxocara IgG antibodies in this analysis of a large CDC dataset weighted to be representative of the U.S. population was $16 \%$, similar to other estimates of anti-Toxocara IgG antibody prevalence in the United States, which, however, are also based on NHANES III data (Lee et al. 2014). Prevalence varied with gender, race-ethnicity, educational attainment and PIR, consistent with findings from a review that also found evidence for an association between Toxocara antibodies and gender, race-ethnicity and education (Lee et al. 2014). While our study was not designed to determine the reasons toxocariasis exposure varies between different demographic groups, Toxocara seroprevalence likely depends on risk of exposure, with different groups having different exposure risks. In contrast to some studies, Won et al. (2008) found that dog ownership increased the risk of exposure. Won et al. (2008) also note that lower socioeconomic status can be associated with occupations that increase exposure risk to Toxocara-contaminated soil and can be associated with living in areas with poor sanitation with subsequent contamination with Toxocara eggs. In terms of environment, it has been suggested that soil in public parks, for example, may contain eggs which under ideal conditions can last for months or even years (Fan et al. 2013).

Furthermore, there are clear differences in infection rates based on geography in the United States, with higher prevalences in the South and Northeast that could have an effect by itself but could also potentially interact with race-ethnicity (Congdon and Lloyd 2011). The differences between males and females could also reflect occupational exposure (Congdon and Lloyd 2011). Finally, in addition to behavioural risk factors, prior research has also suggested that there is likely an association between genetics and susceptibility (i.e. parasitic burden) (Quinnell 2003). 
While some prior work has suggested that being very young or very old increase the risk of infection with toxocariasis and other studies have shown that Toxocara seroprevalence is not necessarily higher for children than for young to middle-aged adults (Won et al. 2008), we found no association with age. In contrast to the finding of an association between toxocariasis seroprevalence and lead concentration in children with higher lead concentrations in the group positive for toxocariasis (Walsh and Haseeb 2012), we did not find an association between seroprevalence and lead concentration in adults. The reasons for this difference are unclear and no studies of which we are aware have addressed the association between blood-lead concentration and toxocariasis in adults. While factors affecting exposure to Toxocara and lead appear to be similar in children (Walsh and Haseeb 2012), they may be different in adults.

In terms of the study's hypothesis, we found a small but significant main multivariate effect for an association between IgG antibodies for Toxocara and cognitive performance in young to middle-aged adults while controlling for age, gender, ethnicity, educational attainment, PIR and blood-lead concentration. Although the multivariate test was significant, the main effect for the association between Toxocara and cognition appeared to be mainly driven by the SDS. The reason why the SDS test appeared to be the most sensitive to the effects of toxocariasis in this study may be because the SDS is a measure of processing speed and not only requires intact response time but performance on the SDS is also influenced by memory (e.g. remembering which symbols are paired with each number on this task reduces completion time because the subject does not need to check the pairs on the screen). In other words, performance on the SDS is influenced by skills necessary to do well on the SRT (i.e. intact simple reaction time) as well as the SDL (i.e. memory), possibly resulting in a more sensitive test of overall brain function. In fact, tasks similar to the SDS (e.g. Digit Symbol from the Wechsler scales, from which the SDS was developed) have been shown to be very sensitive to overall brain function (Lezak et al. 2012). Finally, significant interactions between Toxocara seropositivity and age, gender and educational attainment suggested that risk for decreased cognitive performance is multifactorial in that specific interactions between toxocariasis and demographic variables were associated with decreased cognitive function.

As can be seen in Figure 1, performance on the SDL was in the expected direction (i.e. worse cognitive function associated with Toxocara) for males (note lower scores represent better function) but not females. In fact, although it was a small difference, females positive for Toxocara actually performed better on the SDL than did seronegative females. Thus, these data suggest the possibility of a gender difference, although this was not found in the other two cognitive tests. Notably, the study by Walsh and Haseeb (2012) did not find an effect for gender. In the present study there are a few possibilities that may be related to a gender difference. First, it is possible that there were differences in parasite burden. Unfortunately, the available data did not include serointensity or other measure to determine this; we can only note that the prevalence of Toxocara was higher in males. Second, some studies evaluating the effects of a different parasitic infection on cognition in humans, apicomplexan Toxoplasma gondii, have reported gender differences (Flegr et al. 2012), although others have not (Gale et al. 2015). Finally, gender differences associated with the brain's immune response could be a factor (Lenz and McCarthy 2015).

Recent studies have demonstrated an association between seropositivity for several infectious diseases and cognitive outcome in otherwise healthy adults (Katan et al. 2013). Moreover, there is evidence that infectious disease may contribute to the development or progression of dementia (Malaguarnera et al. 2004). Our findings suggest that toxocariasis is also associated with decreased cognitive function in young to middle-aged adults, although with the exceptions we have described. This finding is significant because toxocariasis is preventable but appears to have effects that persist long after acute infection. In fact, Walsh and Haseeb (2012) suggest that it may be difficult to distinguish between the effects of parasitic infections such as toxocariasis and chronic disease because of persisting effects of the infection.

Because toxocariasis in the current study was detected through anti-Toxocara IgG antibodies, indicating only exposure to Toxocara, we could not determine when the original infection occurred, nor could we rule out the possibility of re-infection. Thus, we cannot determine if the effects of toxocariasis on cognition in these adults is reflective of a disruption in brain development or maturation, an on-going neuroinflammatory response negatively impacting cognition, direct injury from the presence of the parasite to specific neural pathways, or a combination of these or other factors. We also could not determine whether time since the initial infection affects cognition. Insight into how Toxocara might affect cognitive function could be gained from prior work linking toxocariasis with brain disorders such as epilepsy (Quattrocchi et al. 2012) and toxocariasis and toxoplasmosis with psychiatric disorders such as schizophrenia (Khademvatan et al. 2014).

While the exact mechanisms resulting in damage to the central nervous system (CNS) in toxocariasis are unclear, there are several possibilities. For example, toxocariasis can cause significant CNS infection resulting in conditions such as encephalitis, although encephalitis from toxocariasis is uncommon (Finsterer and Auer 2013). In fact, most persons seropositive for toxocariasis are asymptomatic and do not demonstrate overt neurologic signs (Finsterer and Auer 2007). However, studies in animal models have demonstrated significant biochemical changes following experimental infection with Toxocara, including increased expression of brain-injury biomarkers (Liao et al. 2008), up-regulation of pro-inflammatory cytokines (Holland and Hamilton 2013), and alterations in neurotransmitter levels including reductions in gamma-aminobutyric acid, dopamine and serotonin, and an increase in norepinephrine (Othman et al. 2010). 
Our results extend the findings of Walsh and Haseeb (2012), who found decreased cognitive function in children ages 6 to 16 who were seropositive for Toxocara. We cannot compare our results directly to those of Walsh and Haseeb (2012) because different cognitive tests were administered to children than to the adults enrolled in the NHANES III survey. While some prior work has suggested that being young or old increases the risk of infection with toxocariasis, other studies have shown that Toxocara seroprevalence is not necessarily higher for children than for young to middle-aged adults (Won et al. 2008). We found no association with age.

Because prior work and most of the current findings are consistent with negative changes in behaviour or cognition associated with parasitic infection, our finding that one aspect of cognitive function, reaction time as measured by the SRT, remains stable in adults seropositive for Toxocara as shown in Figure 3 is counterintuitive. It is unclear if Toxocara may be protective, or at least not detrimental, to reaction time in adults after age 40 . Though unusual, the possibility that the Toxocara spp. infection may be beneficial is not without precedent, at least in animal models and in certain situations. For example, Queiroz et al. (2013) infected rats with either Toxoplasma gondii or Toxocara canis, or both, and found that either infection alone affected behaviour but that those rats infected with both were indistinguishable from control rats, a finding suggesting that the combination of T. gondii and Toxocara was protective against cognitive decline.

Similarly, Correa et al. (2014) performed a parallel study in mice and found that although mice infected with $T$. gondii had worse learning and memory compared to controls, mice with both $T$. gondii and Toxocara performed normally as did mice only infected with Toxocara. It is unclear if a similar effect would be seen in humans. It is also unclear what the underlying mechanism of such an effect might be. However, the effects of Toxocara on neurotransmitter function and in particular Toxocara's association with increased norepinephrine transmission (Othman et al. 2010) may affect cognition, in that norepinephrine has been associated with cognitive function (Zhang et al. 2013). Finally, due to limitations in the current dataset, it is possible that the finding illustrated in Figure 3 is simply an artefact, possibly related to group differences in length of time with infection. Unfortunately, there is no way to further investigate these findings from the dataset we used.

Strengths of this study include its reliance on a large dataset weighted to represent the United States' population. The exposure variable was based on a laboratory assay and the outcome variables were based on objective neuropsychological tests, factors that reduced the potential for recall or ascertainment bias. Further, we controlled for a variety of sociodemographic variables to diminish confounding, although residual confounding could be present in that there may be relevant variables for which we were unable to adjust. We also evaluated the effects of potential interactions on cognitive functioning between toxocariasis and several variables.

Several additional limitations of this study require consideration. We cannot determine from these data when the original infection occurred and there could be an association between cognition and length of infection or age at time of infection. The cross-sectional design precludes establishing a causal relationship, so it is unclear whether the infection or the cognitive impairment came first. Although we think that the infection likely leads to the cognitive impairment, to fully answer this question would require detailed information regarding time of infection as well as pre-infection and post-infection longitudinal analyses. Finally, while the cognitive measures included have been demonstrated to be reliable and valid, they are fairly cursory and only address limited cognitive domains.

In conclusion, in this large NHANES III-based study, toxocariasis appeared to be associated with decreased cognitive function in adults 21-59 years of age in a controlled multivariate model. Interactions between toxocariasis and age, gender and educational attainment on cognitive function suggest further that certain groups may be more susceptible to the cognitive dysfunction associated with toxocariasis than other groups.

\section{REFERENCES}

Aiello A.E., HaAn M., Blythe L., Moore K., Gonzalez J.M., JAGUST W. 2006: The influence of latent viral infection on rate of cognitive decline over 4 years. J. Am. Geriatr. Soc. 54: 1046-1054.

Arcia E., Otтo D.A. 1992: Reliability of selected tests from the neurobehavioral evaluation system. Neurotoxicol. Teratol. 14: 103-110.

Baker E.L., Letz R.E., Fidler A.T., Shalat S., Plantamura D., LYNDON M. 1985: A computer-based neurobehavioral evaluation system for occupational and environmental epidemiology: methodology and validation studies. Neurobehav. Toxicol. Teratol. 7: 369-377.

Beydoun M.A., Beydoun H.A., Shroff M.R., Kitner-Triolo M.H., Zonderman A.B. 2013: Helicobacter pylori seropositivity and cognitive performance among US adults: evidence from a large national survey. Psychosom. Med. 75: 486-496.

Centers for Disease Control and Prevention 2007: National Center for Health Statistics (NCHS). National Health and Nutrition Examination Survey Questionnaire (Laboratory Pro- tocol). Hyattsville, MD: U.S. Department of Health and Human Services, Centers for Disease Control and Prevention, ftp://ftp. cdc.gov/pub/Health_Statistics/NCHS/nhanes/ nhanes3/26a/SSTOXO.pdf.

Chieffi P.P., Aquino R.T., Pasqualotti M.A., Ribeiro M.C., Nasello A.G. 2010: Behavioral changes in Rattus norvegicus experimentally infected by Toxocara canis larvae. Rev. Inst. Med. Trop. São Paulo 52: 243-246.

Congdon P., Lloyd P. 2011: Toxocara infection in the United States: the relevance of poverty, geography and demography as risk factors, and implications for estimating county prevalence. Int. J. Publ. Hlth. 56: 15-24.

Correa F.M., Chieffi P.P., Lescano S.A., Santos S.V. 2014: Behavioral and memory changes in Mus musculus coinfected by Toxocara canis and Toxoplasma gondii. Rev. Inst. Med. Trop. São Paulo 56: 353-356.

Despommier D. 2003: Toxocariasis: clinical aspects, epidemiology, medical ecology, and molecular aspects. Clin. Microbiol. Rev. 16: 265-272. 
FAN C.K., Liao C.W., Cheng Y.C. 2013: Factors affecting disease manifestation of toxocarosis in humans: genetics and environment. Vet. Parasitol. 193: 342-352.

Fillaux J., Magnaval J.F. 2013: Laboratory diagnosis of human toxocariasis. Vet. Parasitol. 193: 327-336.

Finsterer J., Auer H. 2007: Neurotoxocarosis. Rev. Inst. Med. Trop. São Paulo 49: 279-287.

Finsterer J., Auer H. 2013: Parasitoses of the human central nervous system. J. Helminthol. 87: 257-270.

Flegr J., Guenter W., Bielinski M., Deptula A., ZalasWiecek P., Piskunowicz M., Szwed K., Bucinski A., Gospodarek E., Borkowska A. 2012: Toxoplasma gondii infection affects cognitive function - corrigendum. Folia Parasitol. 59: $253-254$

Gale S.D., Brown B.L., Erickson L.D., Berrett A., HedgES D.W. 2015: Association between latent toxoplasmosis and cognition in adults: A cross-sectional study. Parasitology 142: $557-565$.

Hamilton C.M., Stafford P., Pinelli E., Holland C.V. 2006 A murine model for cerebral toxocariasis: characterization of host susceptibility and behaviour. Parasitology 132: 791-801.

Havlíček J., Gašová Z., Smith A.P., Zvára K., Flegr J. 2001: Decrease of psychomotor performance in subjects with latent 'asymptomatic' toxoplasmosis. Parasitology 122: 515-520.

Holland C.V., Hamilton C.M. 2013: The significance of cerebral toxocariasis: a model system for exploring the link between brain involvement, behaviour and the immune response. J. Exp. Biol. 216: 78-83.

Katan M., Moon Y.P., Paik M.C., Sacco R.L., Wright C.B., ELKIND M.S. 2013: Infectious burden and cognitive function: the northern Manhattan study. Neurology 80: 1209-1215.

Khademvatan S., Khajeddin N., Izadi S., Yousefi E. 2014: Investigation of anti-Toxocara and anti-Toxoplasma antibodies in patients with schizophrenia disorder. Schizophrenia Res. Treatm. 2014: 230349 .

Kountouras J., Tsolaki M., Gavalas E., Boziki M., Zavos C., Karatzoglou P., Chatzopoulos D., Venizelos I. 2006: Relationship between Helicobacter pylori infection and Alzheimer disease. Neurology 66: 938-940.

Krieg E.F., Jr., Chrislip D.W., Letz R.E., Otto D.A., Crespo C.J., Brightwell W.S., Ehrenberg R.L. 2001: Neurobehavioral test performance in the third national health and nutrition examination survey. Neurotoxicol. Teratol. 23: 569-589.

Kusbeci O.Y., Miman O., Yaman M., Aktepe O.C., Yazar S. 2011: Could Toxoplasma gondii have any role in Alzheimer disease? Alzheimer Dis. Assoc. Disord. 25: 1-3.

Lee R.M., Moore L.B., Bottazzi M.E., Hotez P.J. 2014: Toxocariasis in North America: a systematic review. PLoS Negl. Trop. Dis. 8: e3116.

Lenz K.M., McCarthy M.M. 2015: A starring role for microglia in brain sex differences. Neuroscientist 21: 306-321.

Lezak M.D., Howieson D.B., Bigler E.D., Tranel D. 2012 Neuropsychological Assessment. New York, Oxford University Press, 1200 pp.
Liao C.W., Fan C.K., Kao T.C., Ji D.D., Su K.E., Lin Y.H., Сно W.L. 2008: Brain injury-associated biomarkers of tgf-beta1, s100b, gfap, nf-l, ttg, abetapp, and tau were concomitantly enhanced and the ups was impaired during acute brain injury caused by Toxocara canis in mice. BMC Infect. Dis. 8: 84 .

Lurain N.S., Hanson B.A., Martinson J., Leurgans S.E., Landay A.L., Bennett D.A., Schneider J.A. 2013: Virological and immunological characteristics of human cytomegalovirus infection associated with Alzheimer disease. J. Infect. Dis. 208: 564-572.

Malaguarnera M., Bella R., Alagona G., Ferri R., Carnemolla A., Pennisi G. 2004: Helicobacter pylori and Alzheimer's disease: A possible link. Eur. J. Intern. Med. 15: 381-386.

Marmor M., Glickman L., Shofer F., Faich L.A., Rosenberg C., Cornblatt B., Friedman S. 1987: Toxocara canis infection of children: epidemiologic and neuropsychologic findings. Am. J. Publ. Hlth. 77: 554-559.

Othman A.A., Abdel-Aleem G.A., Saied E.M., Mayah W.W., Elatrash A.M. 2010: Biochemical and immunopathological changes in experimental neurotoxocariasis. Mol. Biochem. Parasitol. 172: 1-8.

Quattrocchi G., Nicoletti A., Marin B., Bruno E., Druet-Cabanac M., Preux P.M. 2012: Toxocariasis and epilepsy: systematic review and meta-analysis. PLoS Negl. Trop. Dis. 6: e1775.

Queiroz M.L., Viel T.A., Papa C.H., Lescano S.A., Chieffi P.P. 2013: Behavioral changes in Rattus norvegicus coinfected by Toxocara canis and Toxoplasma gondii. Rev. Inst. Med. Trop. São Paulo 55: 51-53.

QuinNell R.J. 2003: Genetics of susceptibility to human helminth infection. Int. J. Parasitol. 33: 1219-1231.

Schwartz B.S., Stewart W.F., Bolla K.I., Simon P.D., Bandeen-Roche K., Gordon P.B., Links J.M., Todd A.C. 2000: Past adult lead exposure is associated with longitudinal decline in cognitive function. Neurology 55: 1144-1150.

Sturchler D., Weiss N., Gassner M. 1990: Transmission of toxocariasis. J. Infect. Dis. 162: 571.

Suresh S., Sabanayagam C., Shankar A. 2011: Socioeconomic status, self-rated health, and mortality in a multiethnic sample of US adults. J. Epidemiol. 21: 337-345.

Walsh M.G., Haseeb M.A. 2012: Reduced cognitive function in children with toxocariasis in a nationally representative sample of the United States. Int. J. Parasitol. 42: 1159-1163.

Won K.Y., Kruszon-Moran D., Schantz P.M., Jones J.L. 2008: National seroprevalence and risk factors for zoonotic Toxocara spp. Infection. Am. J. Trop. Med. Hyg. 79: 552-557.

Zhang Z., Cordeiro Matos S., Jego S., Adamantidis A., Seguela P. 2013: Norepinephrine drives persistent activity in prefrontal cortex via synergistic alpha1 and alpha2 adrenoceptors. PLoS ONE 8: e66122. 